\title{
Los niveles de ruido en una biblioteca universitaria, bases para su análisis y discusión
}

\author{
Faustino Moreno Ceja \\ Martha Georgina Orozco Medina \\ María del Rocío Zumaya Leal *
}

Artículo recibido:

17 de septiembre de 2013.

Artículo aceptado:

4 de septiembre de 2014.

\section{Resumen}

El presente trabajo determina los niveles de ruido ambiental en una biblioteca universitaria. Se consideraron 10 puntos estratégicos en el interior de las instalaciones y en tres periodos diferentes del calendario escolar. La medición utilizó un sonómetro CESVA 160. Se obtuvieron registros por encima de los 60 decibeles y máximos cercanos a los 70, valores que resultaron fuera del intervalo recomendado por la Organización Mundial de la Salud. Las áreas de mayor ruido fueron la Sala de lectura y el área del mostrador de Circulación. Las principales fuentes de ruido identificadas fueron los usuarios, aviones, teléfonos celulares y equi-

* Los autores pertenecen a la Universidad de Guadalajara, México. (fmoreno@cucba. udg.mx); (mgeorozco@gmail.com); (rzumaya@cucba.udg.mx)

INVESTIGACIÓN BIBLIOTECOLÓGICA, Vol. 29, Núm. 66, mayo/agosto, 2015, México,
ISSN: 0187-358X. pp. 197-224 
pos de cómputo. Se señala como factor crítico el diseño arquitectónico del edificio, en virtud del efecto de reverberación que genera, el cual determina la pobre calidad acústica de las instalaciones. Se concluye que el profesional de la información debe participar en el diseño arquitectónico y que hay que fomentar la cultura bibliotecaria, además de generar y aplicar normas y estándares pertinentes.

Palabras clave: Ruido; Bibliotecas universitarias; Universidad de Guadalajara; Usuarios; Contaminación acústica.

\section{Abstract}

\section{Bases for analysis and discussion of noise levels in a} university library

Faustino Moreno-Ceja; Martha Georgina Orozco-Medina and María del Rocío Zumaya-Leal

This paper examines ambient noise levels in a university library, taking measurements at ten key locations with in the library installation over three periods of the school calendar, using a CESVA 160 sound level meter. Decibel levels above 60 and several touching 70 were recorded, the latter of which surpasses World Health Organization recommendations. The reading room and the checkout desk were the areas exhibiting the highest noise levels. The main sources of noise identified were users, passing aircraft, mobile telephones and computer equipment. A critical factor in these noise levels is the inadequate acoustic design of the library building provoking excessive reverberation. Researchers conclude that information and library professionals should participate in the design of buildings to be used as libraries. Moreover, pertinent stakeholders should promote library culture through the issuance of and enforcement of standards.

Keywords: Noise; University Libraries; Universidad de Guadalajara; Users; Noise pollution. 


\section{INTRODUCCIÓN}

Se ha escrito mucho acerca de la importancia de diversos aspectos inheS rentes a las bibliotecas, como su administración, el manejo de la información, su misión y los servicios que presta; así como los parámetros de calidad, el uso de nuevas tecnologías, los usuarios, su construcción, etc. Para Muñoz (2004: cuarta de forros), "[...] las bibliotecas son la expresión de la cultura de una sociedad”; pero también señaló que en cada época se construyeron bibliotecas según la propia visión del universo, y se creó un microcosmos que reflejaba y resumía el mundo y hacía, al mismo tiempo, un intento por abarcar, mediante el orden y las clasificaciones, un saber heterogéneo e imperfecto. De este modo, las bibliotecas son el resultado de esa tensión entre un conocimiento incompleto y desordenado y la representación del mundo como manifestación del orden, la regularidad y la perfección. Termina Muñoz diciendo que al evolucionar en el tiempo la imagen del universo y las interconexiones de las ciencias humanas, los modelos de la biblioteca cambiaron, lo cual es un reflejo de la estructura del saber y de la forma de organizar la mente humana.

Hoy día se habla de una nueva generación: los "nacidos en la era digital". Según Tapscott (1998), son aquellos cuyo nacimiento fue posterior a 1982. Por otra parte, Prensky (2001a y b) sostuvo que éstos sufrieron cambios, no sólo superficiales sino que también modificaron su forma de hablar o su vestuario a diferencia de generaciones anteriores, "singularidad" que fue causada por el profundo impacto que generó la introducción de la tecnología digital en su vida cotidiana. Representan la primera generación que creció rodeada de tecnologías, tales como la Internet, los videojuegos o los teléfonos móviles y, por consiguiente, poseen ciertas características y habilidades respecto a las Tecnologías de la Información y Comunicación (TIC) y llegan a la biblioteca con una nueva expectativa con respecto al servicio. Sin duda, esto influye de manera significativa en los espacios destinados a la lectura dentro de las instalaciones de ésta (Gisbert y Esteve, 2011).

Dicho lo anterior, cabe decir que debería existir un trabajo conjunto entre el proyecto arquitectónico y los profesionales bibliotecarios al momento de diseñar y construir una biblioteca, de forma que el edificio cuente con buena arquitectura y permita un buen funcionamiento. Sin embargo, a veces sucede, como es el caso del edificio en cuestión, que no se cuenta con espacios aislados en las bibliotecas universitarias que proporcionen un lugar de confort y quietud para los usuarios que requieren de un sitio con estas características. 
$\mathrm{Al}$ ingresar a la biblioteca central del Centro Universitario de Ciencias Biológicas y Agropecuarias (CUCBA) de la Universidad de Guadalajara (UdeG), como usuario o visitante, uno se percata que de manera constante está a su máxima capacidad, y además se observa que el edificio no es utilizado de forma adecuada por ciertos estudiantes y en algunos otros casos tampoco por el propio personal que labora dentro de ella. Ambos actores presentan una escasa o nula cultura bibliotecaria, es decir, el conocimiento de todo lo concerniente al libro y por consiguiente al lugar donde se tienen ordenados éstos -la biblioteca- o bien, donde se coleccionan los libros, los documentos, los manuscritos, etc., y todo ello genera una contaminación por ruido, en tanto que están, además de los usuarios y el personal, las computadoras, los equipos de fotocopiado, se oye taconeo y hay reuniones de equipo, celulares y otros sonidos.

Es por eso que surge el interés por conocer las condiciones acústicas del edificio de la biblioteca central del CUCBA, donde se atiende en promedio a 600 usuarios por día. Así, se llevó a cabo una revisión exhaustiva de la literatura disponible y, sorprendentemente, se encontraron pocas investigaciones relacionadas con el tema.

\section{Antecedentes}

Desde tiempos remotos el ruido ha sido sin duda un problema ambiental para el ser humano. Aproximadamente 600 años a. de n. e., en la ciudad de Síbaris en la antigua Italia, se estableció lo que se podría considerar como uno de los primeros ejemplos de norma con relación al ruido en una comunidad, pues los herreros y carpinteros que ahí laboraban fueron reubicados fuera de la ciudad porque el ruido que producían resultaba molesto (German y Santillán, 2006).

En este mismo sentido, en la antigua Roma (753 a. de n. e.) se prohibió por la noches el tránsito para controlar el ruido emitido por las ruedas de hierro de los vagones que golpeaban las piedras del pavimento y perturbaban el sueño y molestaban a los romanos. Igualmente en algunas ciudades de Europa medieval no se permitía usar carruajes ni cabalgar durante la noche con la intención de asegurar el reposo de la población (Berglund, Lindvall y Schwella, 1999).

Siguiendo con Berglund, Lindvall y Schwella, los problemas con el ruido del pasado no se comparan con los de la sociedad moderna. Un gran número de autos transitan regularmente por nuestras ciudades y campos, y los camiones de carga pesada con motores diésel sin silenciadores adecuados circulan 
por ciudades y carreteras día y noche. Las aeronaves y trenes también contribuyen al ruido ambiental. E igualmente la industria y la maquinaria emiten altos niveles de ruido, así como los centros de esparcimiento y juegos perturban la tranquilidad de las poblaciones.

Las fuentes de ruido se relacionan entonces con el transporte, la actividad laboral e industrial, la recreación y todo el conocido ruido comunitario propio de la concentración de personas. Los actuales estudios de ruido ambiental han cobrado significativa importancia debido a los efectos que tienen en la salud de las personas y las implicaciones económicas y laborales que representan (Orozco, 2008).

\section{Definición de ruido}

Berglund, Lindvall y Schwella (1999) señalan que físicamente no existe ninguna distinción entre sonido y ruido. El sonido es una sensación sensorial, mientras que el complejo patrón de ondas sonoras se denomina ruido, música, habla, etc. El ruido es considerado como un sonido no articulado, que en función de su intensidad y frecuencia puede causar molestias y en algunos casos afectaciones a la salud (Correa, Osorio y Patiño, 2011).

En Quimbiulco (2008) el ruido se define como una combinación desordenada de sonidos que produce una sensación desagradable que puede ser dañina fisiológicamente para el oído del ser humano.

Otra definición que toma el punto de vista físico sostiene que el ruido es un fenómeno sonoro formado por vibraciones (periodos, ciclos o Hertz) y amplitudes producidas por distintos timbres, dependiendo del material que los origina. Lo esencial del ruido es que se trata de uno de los diversos sonidos que pueden producir efectos fisiológicos, psicológicos y sociales no deseados y molestos para las personas o grupos de personas (Martínez, 2005).

De las fuentes de ruido en las ciudades, los vehículos motorizados son responsables de alrededor del $70 \%$, y de éste, el mayor aporte en intensidad lo representan los vehículos de mayor tamaño, entre ellos la locomoción colectiva. Un segundo grupo lo constituyen las fuentes fijas, es decir, las industrias, la construcción, los talleres, los centros de recreación, etc. Los agentes de menor impacto son aquellos que tienen una incidencia esporádica, pero nada despreciable, como los gritos de los niños, los conciertos al aire libre, las ferias y vendedores callejeros, los sonidos de animales domésticos, los fuegos artificiales, entre otros (Mosca, 1980).

Sanz (1990) clasifica el ruido en cuatro clases dependiendo de su duración: 
- Ruido continuo: cuando los niveles de presión acústica y el espectro de frecuencias varían en función del tiempo en pequeños márgenes. Suele ser originado por máquinas con cargas estables como motores eléctricos o bombas de agua que generan el ruido ambiental de fondo.

- Ruido fluctuante: varía de forma aleatoria en función del tiempo en un margen más o menos grande. En función de la repetición del ruido puede ser periódico o no.

- Ruido transitorio: aquél cuyo nivel sonoro comienza y termina dentro de un periodo de tiempo más o menos largo, como el producido por el paso de un tren o un avión.

- Ruido de impacto: se trata de un incremento brusco y de corta duración del nivel de presión acústica, como el disparo de una pistola. Es un caso especial de ruido transitorio. Además, no se puede olvidar el ruido de fondo que es el existente en ausencia de un ruido perturbador.

De Esteban (2003) sostiene que para medir un sonido (ruido) se deben tener en cuenta tres magnitudes importantes relacionadas con su "agresividad". En primer lugar está la intensidad, es decir, su "nivel", el cual está asociado con la cantidad de energía empleada para generarlo y que se mide en decibeles $(\mathrm{dB})$, adicionalmente si se quiere hacer alusión a la respuesta del oído humano es apropiado referir dBA; luego viene la "frecuencia" de exposición del ruido, y al último está la "duración" del mismo. Para conocer el nivel de contaminación del ruido se deben tener en consideración estas tres magnitudes.

\section{Normatividad}

Los ruidos y vibraciones son, pues, los principales causantes de la contaminación acústica. En la medida en que ésta se constituye como un sinónimo de molestia y provoca una disminución de la calidad de vida de los habitantes de nuestras ciudades, es deber de la Administración establecer lineamientos y normas precisas que permitan controlar estas molestias y garantizar los intereses legítimos de los ciudadanos (Abad et al., 2011).

También es importante tener en cuenta que la calidad acústica en los espacios de desarrollo humano es una responsabilidad compartida, puesto que en la medida en la que los propios ciudadanos se impliquen en disminuir la emisión de actividades ruidosas se respetarán los espacios y se tendrán menos problemas de contaminación por ruido.

Varios países han tratado de atender este problema por medio de la legislación, y ya han determinado los diferentes niveles de ruido según el tipo de 
recinto que los emite; en Europa se ha avanzado notablemente en la caracterización de estas zonas de ruido y estudios de molestia relacionada con la exposición a él. Sin embargo, en muchos otros países, como en México, son pocos los esfuerzos que se han hecho para tomar en cuenta los estudios de ruido como una herramienta de diagnóstico, control y seguimiento (López, s. a.).

La norma mexicana NOM-081-SEMARNAT-1994. Que establece los límites máximos permisibles de emisión de ruido de las fuentes fijas y su método de medición, al señalar que durante el día es de 68 dBA y de noche de 65 dBA, aunque para el Distrito Federal, desde septiembre del año 2006, la norma es más estricta, ya que reduce estos límites a 65 y $62 \mathrm{dBA}$, de acuerdo con la Norma Ambiental NADF-005-AMBT-2006 (Estrada, 2010).

El ruido en la biblioteca es parte del ambiente; sin embargo, la OMS señala que no debe exceder los 45 decibeles. No obstante las dinámicas actuales a las que se someten las bibliotecas universitarias, el ruido se ha convertido en una enorme distracción para aquellos que ven en estos recintos un sitio donde hay paz, tranquilidad y armonía para revisar materiales y estudiar (Crumpton, 2007).

Es importante señalar que los niveles permisibles de ruido son aquellos expresados en el número de decibeles cuya exposición resulta molesta al oído humano. Estos son de gran importancia para determinar la existencia de contaminación y la necesidad de adoptar las medidas técnicas preventivas en busca del bienestar para los usuarios de una biblioteca. En este sentido, las colecciones consideradas de mayor demanda deben colocarse en los espacios donde sea posible tener la vigilancia y el control que influyen y determinan la disminución del ruido.

La normatividad existente para bibliotecas es escasa. En Brasil, por ejemplo, se rigen con la norma NC53-75:82. Bibliotecas públicas y escolares. Especificaciones del proyecto. Establece que el nivel de ruido será inferior a 40 dBA (Quintero y Muñoz, 2009: s. pág.).

Por su parte, Carrión (1998) describió que la curva recomendada de nivel de ruido para despachos y bibliotecas NC es de 30-35, misma que convertida a dBA se sugiere que sea entre 42-46 dBA. En este mismo sentido Quimbiulco (2008) y Bau (2011) señalan que para salas de despachos, bibliotecas y salas de justicia lo recomendable sería fijar el nivel entre 30-40 dBA.

Los Estándares para bibliotecas universitarias chilenas (2001) señalan que el ruido en las salas de lectura dentro de las bibliotecas debe fluctuar entre los 30 y los $50 \mathrm{dBA}$.

El estudio de Ntui (2009) da cuenta de los trabajos llevados a cabo en instancias universitarias, edificios históricos y las bibliotecas y documenta altos 
niveles de ruido que superan la norma establecida para estos recintos. Manifiesta además la necesidad de contar con espacios más aislados para el estudio, la lectura y la comprensión. Resume que hace falta un manejo adecuado para minimizar la contaminación auditiva.

Por último, en el caso de México, las Normas para bibliotecas de instituciones de educación superior e investigación (2012) advierten que dentro de las instalaciones de una biblioteca universitaria el ruido no debe sobrepasar los $50 \mathrm{dBA}$.

No cabe duda que una manera de combatir y evitar la contaminación auditiva es tener conciencia, respeto y consideración hacia los demás, y tratar de que nuestras actividades cotidianas no generen este problema que afecta severamente la salud y la calidad de vida de los otros.

\section{El impacto a la salud}

El impacto que tiene el ruido sobre la salud y el bienestar en los habitantes empieza a manifestarse en mayor medida en las primeras décadas del siglo XX (German y Santillán, 2006) y la preocupación por el ruido como agente contaminante ha llevado a su estudio (Baigorri, 1995). Según la literatura especializada el ruido es considerado como el contaminante más barato de producir y que necesita muy poca energía para ser emitido, aunque también es complejo de medir y cuantificar (Martínez, 2005). Se estima que cerca de un tercio de la población mundial padece de algún grado de sordera o pérdida auditiva causada por exposición a sonidos de elevada intensidad (López et al., 2000).

El estrés o estado de tensión producido por exposición prolongada a ruidos superiores a los $85 \mathrm{dBA}$, por cierto muy común en las ciudades, puede hoy día generar hasta un $12 \%$ más de problemas cardiovasculares, $37 \%$ más de problemas neurológicos y 10\% más de problemas digestivos (García y Garrido, 2003).

De acuerdo con la Organización Mundial de la Salud (OMS)(Berglund, Lindvall y Schwella, 1999) los problemas de salud relacionados con ruido incluyen, entre otros:

- Interferencias con el comportamiento social (agresividad, protesta e impotencia).

- Interferencias con la comunicación oral.

- Disminución en el rendimiento laboral y escolar.

- Dolor y fatiga de la audición.

- Posibles trastornos, disminución o pérdida de la audición.

- Molestias debido a alteraciones en el sueño y que constituyen dificultades en el rendimiento diurno y alteraciones a la respuesta inmune. 
- Efectos cardiovasculares.

- Respuestas hormonales y consecuencias en el metabolismo.

- Alteraciones en la tensión arterial.

- Cambios de conducta en los niños o personas sensibles.

Numerosos estudios han demostrado que existe una relación directa entre el ruido y la salud. Los problemas relacionados con el ruido incluyen enfermedades asociadas con el estrés, la presión arterial alta, la interferencia en la comunicación, los trastornos del sueño, la pérdida de productividad y la pérdida de la audición inducida por ruido (PAIR), éste es tal vez uno de los aspectos que más afectan la salud y el que se discute más a menudo. Según Environmental Protection Agency (EPA), la contaminación acústica afecta negativamente la vida de millones de personas. La Figura 1 pertenece a la Noise Pollution Clearinghouse, la cual hace una comparación entre algunos sonidos comunes y el efecto que producen desde el punto de vista del daño potencial para la audición.

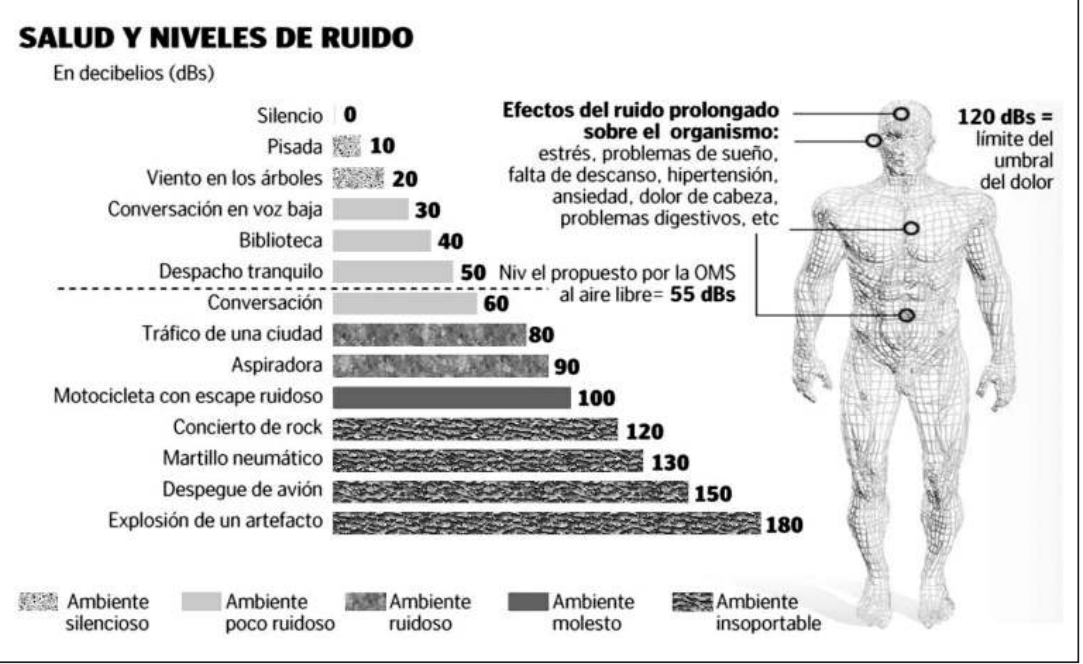

Figura 1. Niveles sonoros y respuesta humana

De Esteban (2003) ha encontrado que la preocupación actual por la protección del medio ambiente se pone de manifiesto en la lucha contra el ruido, dada la atención que prestan los gobiernos de los países desarrollados en promulgar normas que regulen la contaminación sonora de las ciudades. Se estima que en los países de la Unión Europea cerca de 113 millones de personas se encuentran expuestas a niveles de ruido ambiental por encima de los 
65 dBA, límite de tolerancia recomendado por la OMS. España resulta ser el país más ruidoso de Europa y el segundo de la Organización para la Cooperación y el Desarrollo Económico (OCDE) después de Japón.

México ha avanzado en cuanto a la caracterización sonora de espacios laborales a nivel industrial y ha incorporado programas de control sonoro y protección de la audición. En cuanto al nivel de ruido urbano existen algunos esfuerzos aislados, como el mapa de ruido de la zona metropolitana de Guadalajara, y algunos registros en la ciudad de México, Querétaro y Monterrey (Orozco y González, 2012). Lo encontrado en esta investigación identifica la necesidad de impulsar más estudios en este ámbito.

\section{El impacto en la biblioteca}

El silencio en la biblioteca es una necesidad, en virtud de que ésta es considerada como un área propicia para la concentración, la lectura, la consulta y la investigación, en donde las interferencias y distracciones deben ser mínimas. La biblioteca debe contar con espacios libres del bullicio y el ruido, y en los que impere el silencio como norma de respeto dentro del edificio. Se ha determinado al ruido como un distractor que se opone a un espacio tranquilo y amable, factor que lo convierte en un problema de contaminación y salud ambiental.

Se considera que el ruido repentino interfiere de manera directa en el usuario porque provoca una reacción inmediata, que por lo regular conduce a una distracción, la cual potencia la posibilidad de cometer errores y, por lo tanto, afecta la calidad y eficiencia de los trabajos elaborados. Algunas de las principales causas tienen origen en los bibliotecarios de circulación y los propios equipos: impresoras, copiadoras, equipos de aire acondicionado, ventiladores y puertas automáticas, entre otros elementos.

El ruido ambiental es cualquier sonido no deseado que constituye una amenaza para el medio ambiente porque se presenta en el momento y lugar equivocados y suele ser el resultado de la actitud humana, pero también es cada vez mayor con la industrialización, la urbanización y las TIC. El ruido puede causar tensión emocional y convertirse en una fuente de gran frustración cuando está más allá del control de las personas (Ntui, 2009).

Sin embargo, los usuarios también contribuyen de manera significativa a la generación de ruido al no modular su voz, usar estruendosamente los teclados de sus computadoras, generar en su celular continuos sonidos, mover las sillas y mesas intentando generar equipos de trabajo, a lo que se suman las conversaciones y risas con otras personas, además del taconeo inherente de nuestro caminar. 


\section{Aspectos clave para el diseño de una biblioteca}

Este trabajo no intenta evaluar el aspecto arquitectónico de una biblioteca; se centra más bien en la evolución sufrida en el concepto de biblioteca como "guarda de libros y bodega" a ser considerada como un Centro de Recursos para el Aprendizaje y la Investigación (CRAI), como una entidad de "servicio público" cuya misión fundamental es difundir la información que almacena y satisfacer las necesidades del usuario, lo cual nos permite señalar que los edificios deberían sufrir los mismos cambios en los materiales de construcción y adecuar sus espacios para que los usuarios, el personal y los materiales pudieran dejar atrás la antigua estantería cerrada y pasaran a ser espacios abiertos de autoacceso, en los que pudieran también interactuar entre sí.

En este sentido, Martínez (2004) señala que en un nuevo modelo de biblioteca el libro no debe ser el centro de atención, lugar que ahora le corresponde al usuario que aprende. $\mathrm{Al}$ respecto propone cambios en los conceptos espaciales: un edificio que promueva el aprendizaje, que sea accesible y central, tecnológico y digital, abierto y flexible a otros servicios, que sea emblemático, moderno y sostenible. Sin olvidar otras áreas y aspectos, como tener acceso a salas de Internet, aulas informáticas y espacios para la formación de usuarios y el fotocopiado. Este mismo autor señala que "La biblioteca tiende a ser un centro dinamizador del nuevo aprendizaje, en un centro social del campus. La universidad que se arriesgue a optar por realizar estos cambios deberá de transformar la biblioteca a partir de un nuevo planteamiento". (2004: 99)

Ntui (2009) menciona en su estudio que la biblioteca de la universidad es un centro dinámico de la comunidad universitaria que ofrece una amplia gama de servicios y materiales. Fuentes (2005) contribuye con información relevante con el análisis que realiza sobre las bibliotecas universitarias como un entorno híbrido. En este sentido incluimos parte de la descripción que el autor antes mencionado detalla en su documento, en ella considera tres aspectos de la universidad que impactan sustancialmente a la biblioteca: el primero de éstos se refiere al incremento en la matrícula y con ello al número potencial de usuarios; el segundo es el relativo a la evolución en la enseñanza, que es ahora más dinámica, y el tercero involucra a las TIC, y con ello los beneficios, alcances y adecuaciones que éstas representan. También señala que la planificación del edificio debe contemplar los tres elementos principales de la misión de la universidad: la docencia, la enseñanza y la investigación, que deben formar parte de la biblioteca. Destaca también diferentes áreas que deben tener las colecciones, los servicios y la administración, pero sobre todo el cuidado del espacio y las interrelaciones que el trabajo técnico conlleva en la biblioteca. 
Por otro lado, expone que la construcción del edificio debe cuidar elementos como los expuestos en el XII Seminario de la IFLA en el 2001, que se relacionan con la atención a personas con capacidades diferentes, las TIC y la creación de espacios para dar cabida al aprendizaje y la enseñanza. Finalmente, advierte sobre la importancia que tienen los espacios para el personal, la conservación de los materiales y los espacios auxiliares y para publicitar los recursos. También hace alusión a los objetivos que Jean Bleton propone: que el edifico se debe situar en el corazón de la universidad, tener un aspecto arquitectónico significativo y proveer el adecuado confort acústico, visual y de movimiento para los usuarios.

Con base en lo antes señalado es importante considerar previamente a la construcción de una biblioteca elementos como el tipo de biblioteca, el tipo de usuarios a los que atenderá, las instalaciones y el equipamiento. Adicionalmente, hay que tener en cuenta las condicionantes de presupuesto, espacio físico y, por supuesto, la planificación, la elección de tareas entre técnicos y arquitectos; por eso, se reitera la necesidad de participación del profesional de la información, quien puede dar directrices precisas sobre lo que debe ser considerado.

La International Federation Library Association (IFLA) ha convocado por lo menos cinco reuniones para tratar el planeamiento del edificio de la biblioteca: la de 1971 en Lausana, sobre bibliotecas universitarias; la de 1973 en Roma, sobre bibliotecas nacionales; la de 1977 en Bremen, sobre bibliotecas públicas; la de 1980 en Frederiksdal (Dinamarca) sobre el problema de distribución de los espacios, y la de 2001 en Boston, dentro del XII Seminario de la IFLA titulado "Lugares futuros: la reinvención de las bibliotecas en la era digital”, señalada por Fuentes (2005), quien destaca 12 puntos, entre ellos la predicción del crecimiento, el acceso a discapacitados, la seguridad, las telecomunicaciones, el diseño y el confort.

Por otra parte está el ya conocido decálogo del arquitecto inglés Harry que debe asumir todo edificio bibliotecario, descrito en 1973 y actualizado en 1980: flexible, compacto, accesible, extensible, variado, organizado, confortable, seguro, constante y económico. Cabe señalar que estas recomendaciones han sido criticadas por ser demasiado generales (Gómez, 2002); se trata de principios arquitectónicos comunes a todo tipo de construcciones y resultan muy básicos para cualquier edificio; sin embargo, son importantes y deben interpretarse correctamente para llegar a una buena edificación.

En cuanto a las bibliotecas universitarias sólo se diferencian los tipos de puestos de lector según el uso. Bisbrouck (2003) propone cinco zonas funcionales para bibliotecas universitarias, que son fácilmente aplicables a bibliotecas públicas: espacios de entrada, de consulta/trabajo y puesta a disposición de la documentación, de búsqueda de información, para depósitos de libros y para los servicios internos. 
En este mismo tenor es importante mencionar que esto ya se señalaba en la obra Nuevos edificios para las bibliotecas universitarias (Rodríguez, 1996): el concepto estético debe guardar un equilibrio entre la funcionalidad y la flexibilidad entre áreas de servicios, acervo y usuarios, para estudiar e investigar en combinación con la belleza.

\section{OBjetivos}

1. Realizar un estudio de ruido en las instalaciones de la biblioteca central del Centro Universitario de Ciencias Biológicas y Agropecuarias (CUCBA).

2. Determinar los niveles sonoros en los puntos estudiados.

3. Analizar los niveles de ruido registrados en función de los periodos y horarios estudiados.

\section{Metodología}

Al considerar que los niveles de ruido en la biblioteca influyen en la concentración y el estudio, se valoró la posibilidad de realizar un proyecto de investigación que registrara los niveles de presión sonora, por lo cual se eligió la biblioteca central del Centro Universitario de Ciencias Biológicas y Agropecuarias, que tiene una afluencia promedio diaria de 600 usuarios entre estudiantes, profesores y egresados de las carreras de Biología, Ingeniero Agrónomo, Medicina Veterinaria, Agronegocios y Ciencias de los Alimentos, así como diversos posgrados y otros profesionales de áreas afines.

Se realizó una investigación bibliográfica de los estudios relacionados con el ruido en los espacios urbanos y se consultó la normatividad vigente para fuentes fijas (García, 1994; Beristain, 1998; Villalobos y Elizondo, 1999; Chávez, 1998; Delgadillo, 1998; Soriano, 1995; Palafox, 2003, Orozco, 2008 y la NOM-081-SEMARNAT-1994. Que establece los límites máximos permisibles de emisión de ruido de las fuentes fijas y su método de medición). De la información recabada se consideraron algunos criterios para establecer la metodología específica para esta investigación.

- Caracterización del área. El edificio de la biblioteca estudiada se localiza en el camino Ing. Ramón Padilla Sánchez 2100 en el predio las Agujas de Nextipac, Zapopan, Jalisco. Cuenta con una superficie de $1555 \mathrm{~m}^{2}$, de los cuales $466 \mathrm{~m}^{2}$ son ocupados por las áreas del personal, 
$392 \mathrm{~m}^{2}$ por la estantería y los restantes $697 \mathrm{~m}^{2}$ se encuentran divididos en tres salas de lectura, cubículos y sala Wi-Fi para los usuarios. Cuenta además con secciones para procesos técnicos, adquisiciones y mapoteca. El inmueble está construido a una doble altura de $8 \mathrm{~m}$ en su parte central, su estructura es de concreto hidráulico en muros y perfiles estructurales con cristal en fachadas, toda su bóveda fue realizada con estructura metálica y loza-acero, sus muros al interior son aparentes con estructura de aluminio y cristal, y los pisos son de cerámica.

- Selección de puntos de medida. Se seleccionaron 10 puntos de las áreas de la biblioteca en los que destacan los relacionados con los usuarios: tres salas de lectura, los cubículos y la sala de Wi-Fi, y espacios para adquisiciones, procesos técnicos, circulación, acervo de consulta y mapoteca.

- Selección de parámetros, medición y análisis. Respecto al periodo de tiempo establecido para este estudio, se consideró representativo el semestre del calendario escolar, y se tomaron los datos durante la última semana de clases, en periodo de exámenes y al inicio del semestre.

Las mediciones de ruido se realizaron en cada uno de los puntos por lapsos de 5 minutos, conforme las indicaciones del manual del equipo Sonómetro CESVA 160 con ponderación A. Se utilizó el software del equipo para la recuperación de datos, y de éstos se obtuvo un total de 450 registros de nivel sonoro continuo equivalente (Neq, Leq, o NSCE), el cual se define como: "[...] el nivel de energía acústica uniforme y constante que contiene la misma energía que el ruido producido de forma fluctuante por una fuente fija durante el periodo de observación" (NOM-081-SEMARNAT-1994, PUNTO 4.14). Nivel Máximo (Lsmax) y Nivel Mínimo (Lsmin), calculados a partir del total de los registros recuperados. Con base a la siguiente fórmula según la NOM-081-SEMARNAT-1994:

$$
N e q=10 \log \frac{1}{T} \int_{0}^{T} 10_{\frac{10}{N}}^{N} d t
$$

Donde:

Neq $=$ nivel equivalente de cada punto

$N=$ nivel fluctuante para cada punto

$T=$ periodo de observación

En cada punto de medición se colocó el sonómetro en un tripié a $20 \mathrm{~cm}$ del punto a medir y a una altura aproximada de $1.5 \mathrm{~m}$ que simula la altu- 
ra del oído humano, se tomaron tres periodos de medición de 5 minutos cada uno, con filtro de ponderación frecuencia A y filtro de ponderación temporal Fast (rápido). Cada uno de ellos se transfirió a Excel 5.0 para generar las gráficas, analizar los resultados y redactar la interpretación.

\section{Resultados}

Los resultados se presentan en tres fases conforme a los objetivos planteados y a la metodología propuesta. Respecto al origen de los niveles de ruido identificados, destacan el ingreso y la salida del usuario, el sonido que proviene del funcionamiento de impresoras y fotocopiadoras, los teclados de computadoras, las conversaciones entre dos, tres o más usuarios, los teléfonos celulares, los movimientos de sillas y el sonido del impacto de los zapatos y tacones sobre el piso. También eventualmente el paso de aviones influye como fuente de ruido que proviene del exterior, además del producido por las podadoras del recinto, el cual tiene efectos directos en la calidad acústica de la biblioteca.

A continuación se presentan los resultados de las mediciones efectuadas durante los periodos "tipo" que se determinaron para registrar los niveles de presión sonora: al inicio, al fin del semestre y en el periodo de exámenes.

Respecto a los datos de la Figura 2, se observa que los niveles registrados durante el fin de semestre sobrepasan los 50 dBA, y cinco registros están en

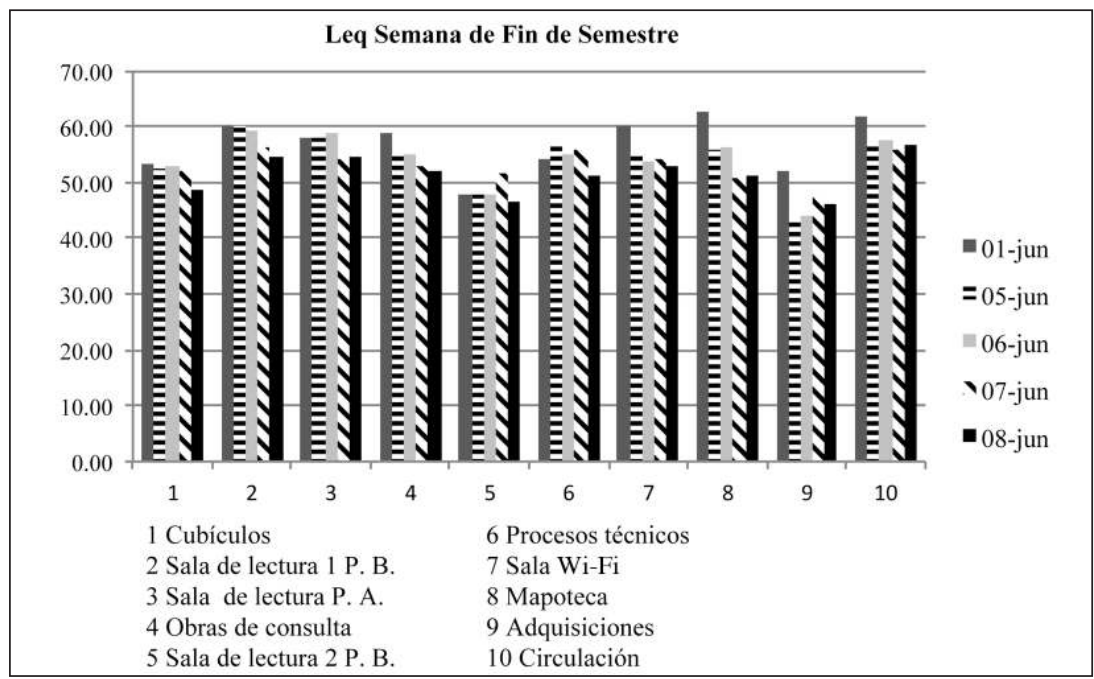

Figura 2. Nivel sonoro de fin de semestre 
$60 \mathrm{dBA}$ o por encima de este rango. Las áreas con mayor intensidad de ruido son las salas de lectura, la de obras de consulta, la mapoteca y la de circulación. El día en que se registró la mayor intensidad fue el lunes y en lo general el día con niveles menores es el viernes, días que coinciden con la variabilidad en la afluencia de estudiantes, motivada por el perfil de los estudiantes (particularmente de Ingeniero Agrónomo y Medicina Veterinaria), dado que existe una significativa cantidad de alumnos foráneos que regresan a sus localidades de origen.

En los resultados de la Figura 3 se observan las mediciones realizadas durante el periodo comprendido en la semana de exámenes; se registró el nivel sonoro continuo equivalente (Leq). Los niveles más elevados se refieren a los puntos 2, 3, 6 y 10, que corresponden a las salas de lectura, procesos técnicos y área de circulación; los que registraron un nivel menor están en los puntos 1,5 y 9 , que son cubículos, sala de lectura 2 de la planta baja y adquisiciones. En esta figura, contrariamente a lo que ocurre en el periodo de clases, la mayor afluencia se tuvo el viernes y por tanto los niveles de ruido se incrementaron, y se destacaron los niveles muy bajos para el día miércoles 13, lo cual sería el ideal que debería registrarse todos los días.

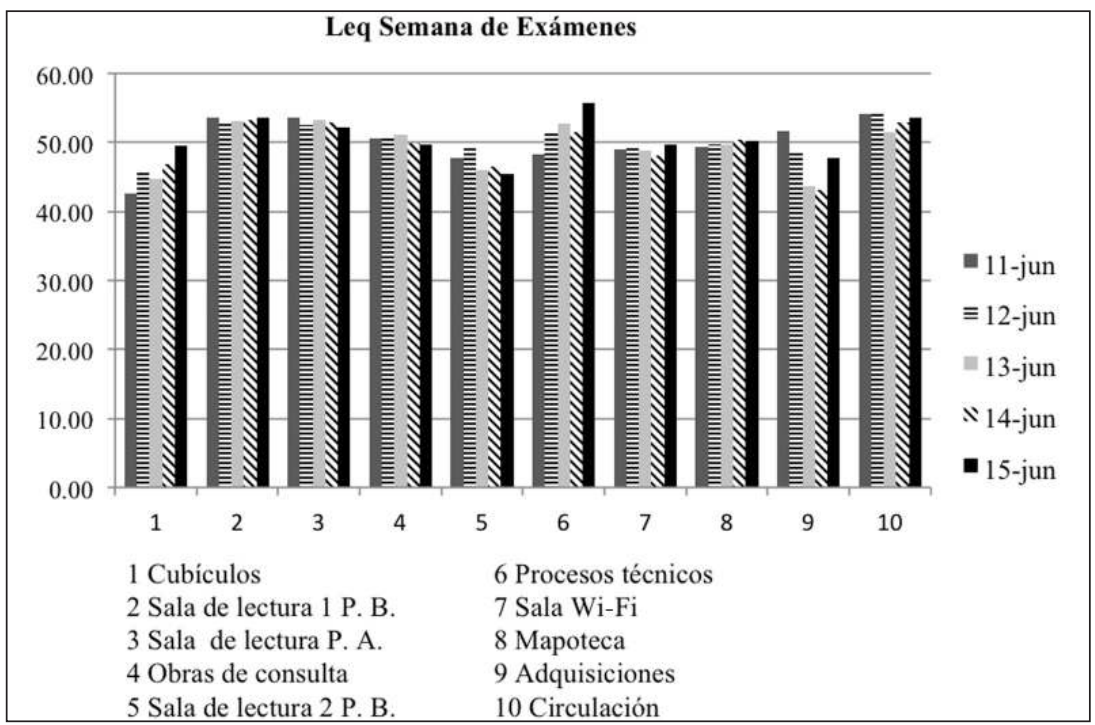

Figura 3. Nivel sonoro en semana de exámenes

En la Figura 4 se observa un notable incremento en los niveles con relación a la semana de exámenes. En los registros que ahora se presentan, se aprecia cómo están próximos al rango de los 60 decibeles, sobresaliendo las áreas de lectura 2 y 3 , y la 10 de circulación. Llama la atención un dato que sobrepasa los 65 
decibeles en la sala de lectura 2 de la planta baja, que es una situación anómala y que está cercana a las regulaciones normativas de nivel máximo admisible en las zonas urbanas en horario nocturno, que son de 68 dBA para el día.

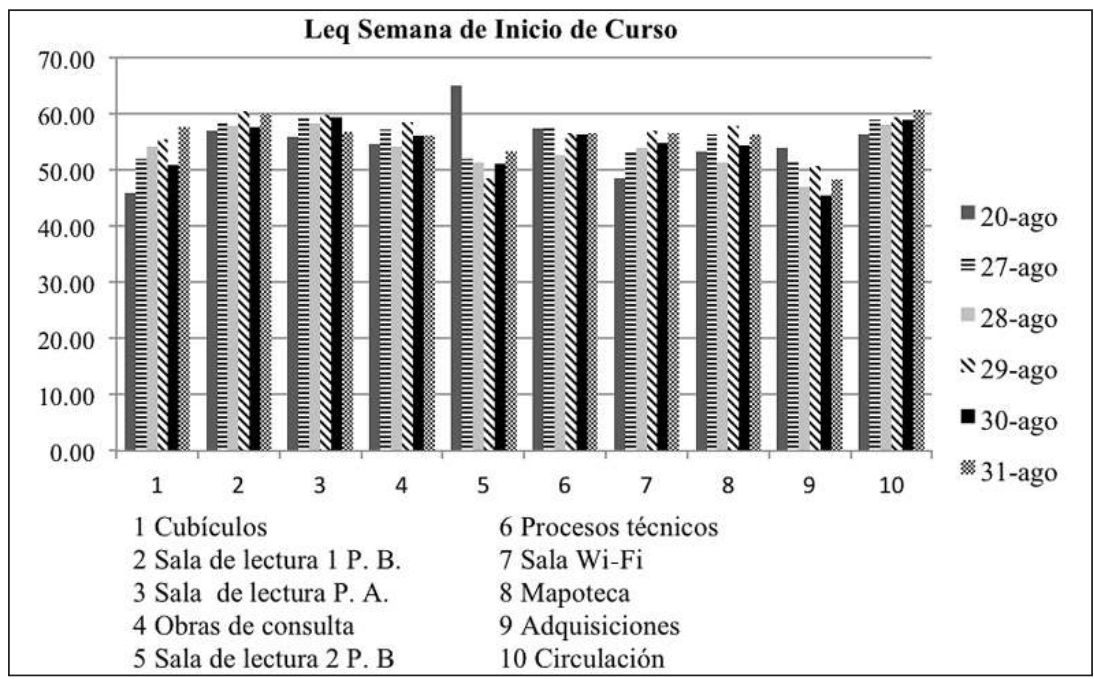

Figura 4. Nivel sonoro inicio de curso semestral

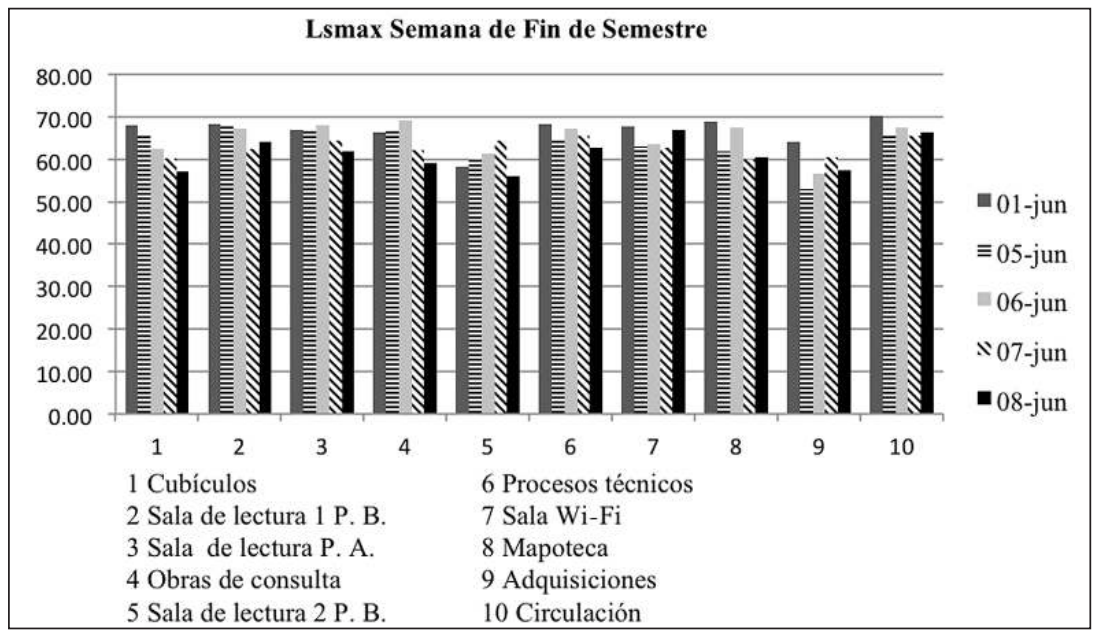

Figura 5. Niveles máximos en fin de semestre

Los niveles máximos en fin de semestre superan los 60 dBA en su mayoría, de acuerdo con los registros presentados en la Figura 5. Éstos deben ser considerados con reserva puesto que se trata de un valor máximo registrado en 
el periodo de 5 minutos de medición, el cual no representa un valor uniforme. Así, los valores encontrados son consecuencia directa de la cantidad de alumnos; sin embargo, el registro en el área de circulación tomado el día 10. de junio rebasó los niveles máximos permisibles dentro de zonas urbanas en horario diurno, como se aprecia en esta figura, que registró hasta los $70 \mathrm{dBA}$.

En la Figura 6 se aprecia que los niveles de presión sonora durante el periodo de medición presentaron variaciones que van desde los $48 \mathrm{dBA}$ hasta los $73 \mathrm{dBA}$. En las áreas de procesos técnicos y circulación se registraron los niveles más altos, atribuidos quizá a la afluencia de personas hacia los sanitarios, y en la Sala Wi-Fi los niveles fueron los de menor intensidad. Sin embargo, como se muestra, todos los niveles están fuera de norma para un recinto bibliotecario de acuerdo con la normativa descrita en este documento en el apartado "Normatividad", con las debidas reservas puesto que se trata de los niveles máximos registrados en el periodo de medición, considerando además que no se trata de valores continuos.

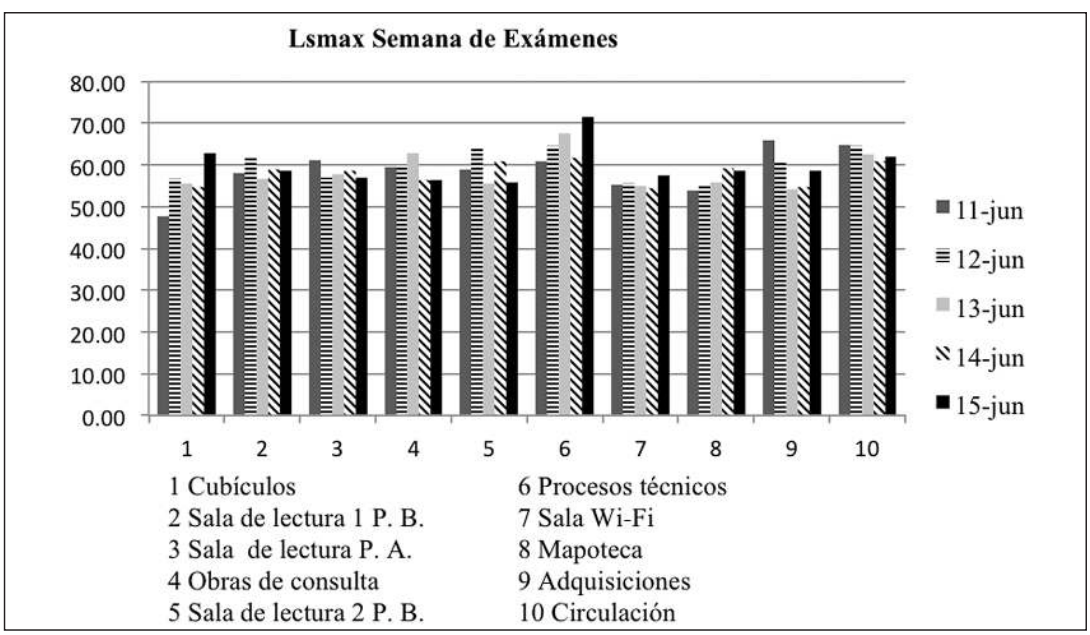

Figura 6. Niveles máximos en semana de exámenes

El nivel máximo registrado se observa en la Figura 7, que corresponde a la semana de inicio de curso; los registros estuvieron muy cercanos a los $70 \mathrm{dBA}$, semejantes a los niveles que se pueden registrar en una avenida a nivel promedio (cabe recordar que un dato similar se presentó en la Figura 4 en esta misma área). Se detectó un registro anómalo de 89 dBA el día 20 en la sala de lectura 2 de la planta baja y otro en el área de adquisiciones; los demás estuvieron en rangos que fluctuaron entre los 60 y $70 \mathrm{dBA}$. En promedio, el lunes fue el día que obtuvo niveles menores de ruido y el viernes los niveles más altos. Es 
importante hacer notar que todos los registros se encuentran fuera de norma para bibliotecas e incluso para las permitidas en zonas urbanas para sonidos provenientes de fuentes fijas, lo que da cuenta de la afluencia de alumnos y lo inadecuado que resulta el edificio, algo ya señalado en otros puntos.

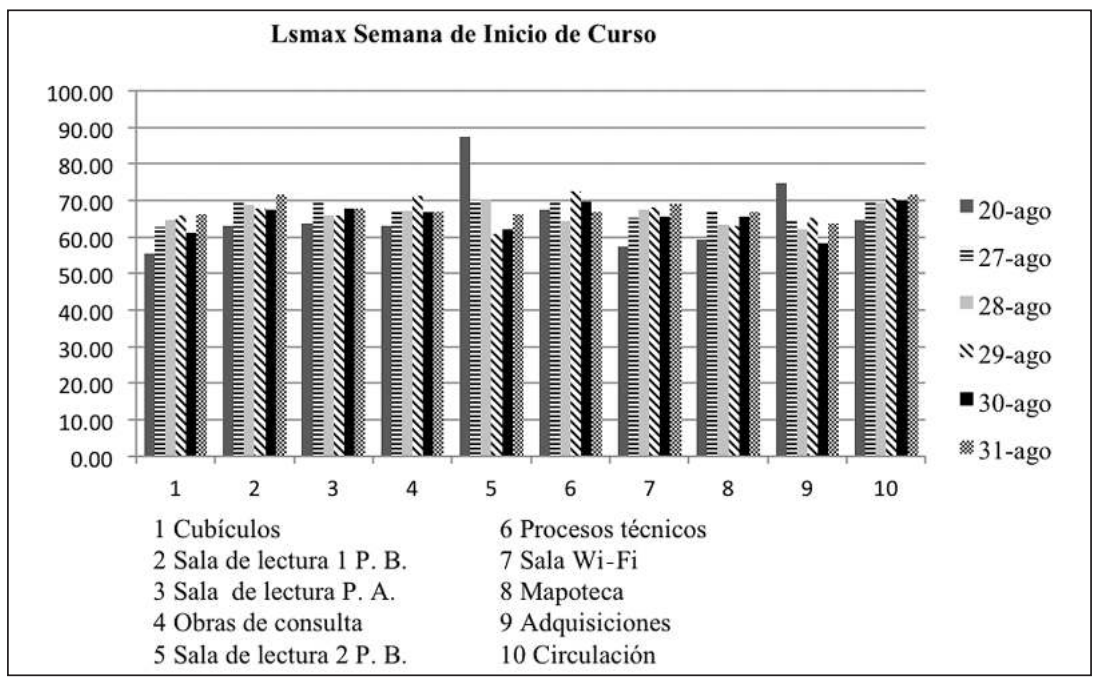

Figura 7. Nivel máximo en inicio de curso semestral

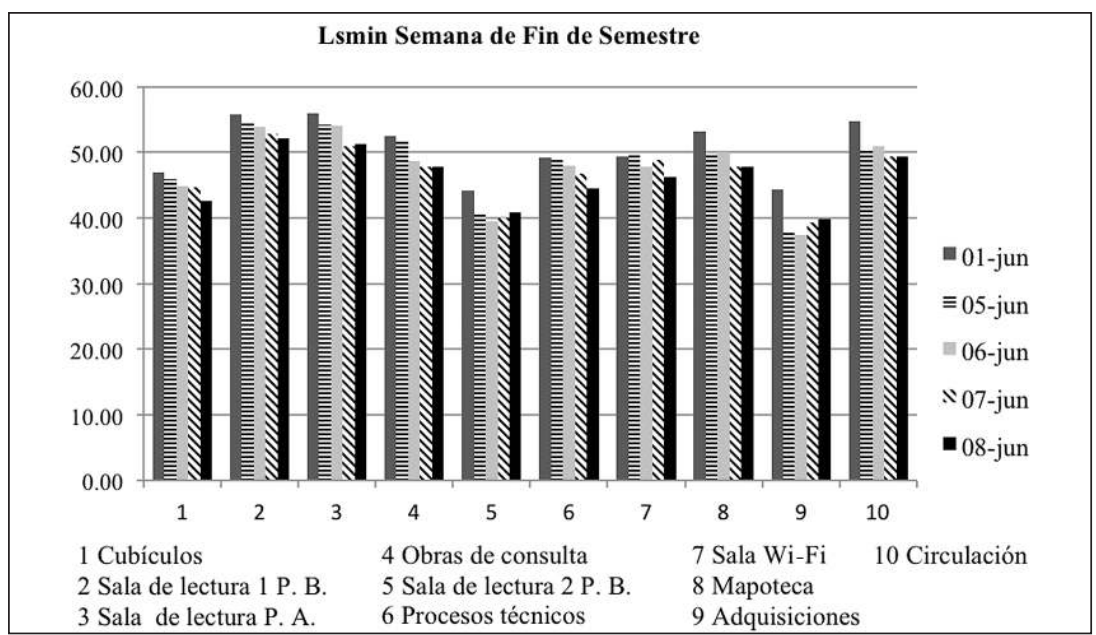

Figura 8. Nivel mínimo en semana de fin de semestre

Los niveles mínimos durante la semana en fin de semestre se presentan en la Figura 8, los cuales están cerca o superan los 50 dBA. En su mayor parte 
no es esta una condición ideal como la que se esperaría encontrar para los niveles mínimos, en virtud de la ausencia de estudiantes; sin embargo, el diseño del edificio deja de manifiesto la inoperatividad, como tal, para una biblioteca, así como la necesidad de incorporar un acondicionamiento acústico que le permita reducir la reverberación, fenómeno que entre otros factores también contribuye al aumento en la intensidad sonora.

De acuerdo con la Figura 9, los datos mínimos en semana de exámenes para los puntos 1, 5 y 9 son semejantes a los encontrados en la figura anterior y dan cuenta de un comportamiento acústico peculiar porque también son áreas en donde se registran los mayores niveles sonoros continuos equivalentes. Destacan las salas de lectura de la planta baja y alta, la de obras de consulta y el área de circulación, con registros muy homogéneos a lo largo de la semana.

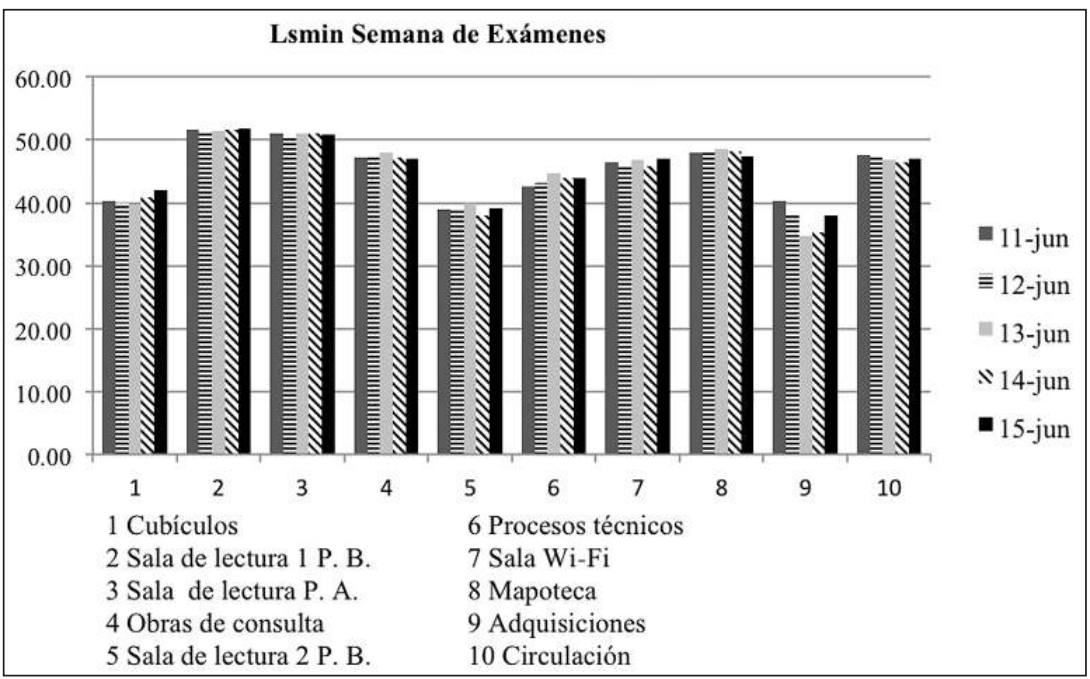

Figura 9. Nivel mínimo en semana de exámenes

El nivel referido como mínimo se muestra en la Figura 10. Al inicio de clases es muy elevado algunos días; por la poca asistencia de alumnos se esperaban niveles de ruido más bajos, esto sólo se pudo observar en tres áreas de la biblioteca, que son el punto 1, los cubículos; el 5, la sala de lectura 2 planta baja, y el 9 , de adquisiciones; el resto de áreas se comporta de manera similar a la de los demás periodos. 


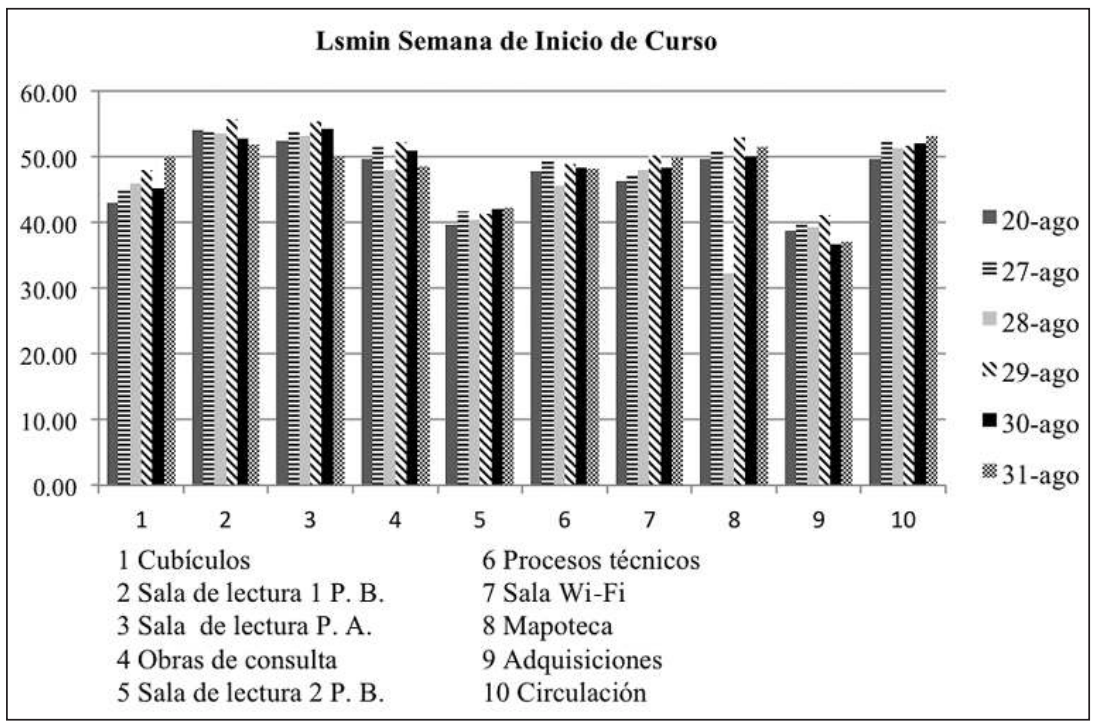

Figura 10. Nivel mínimo en inicio de curso semestral

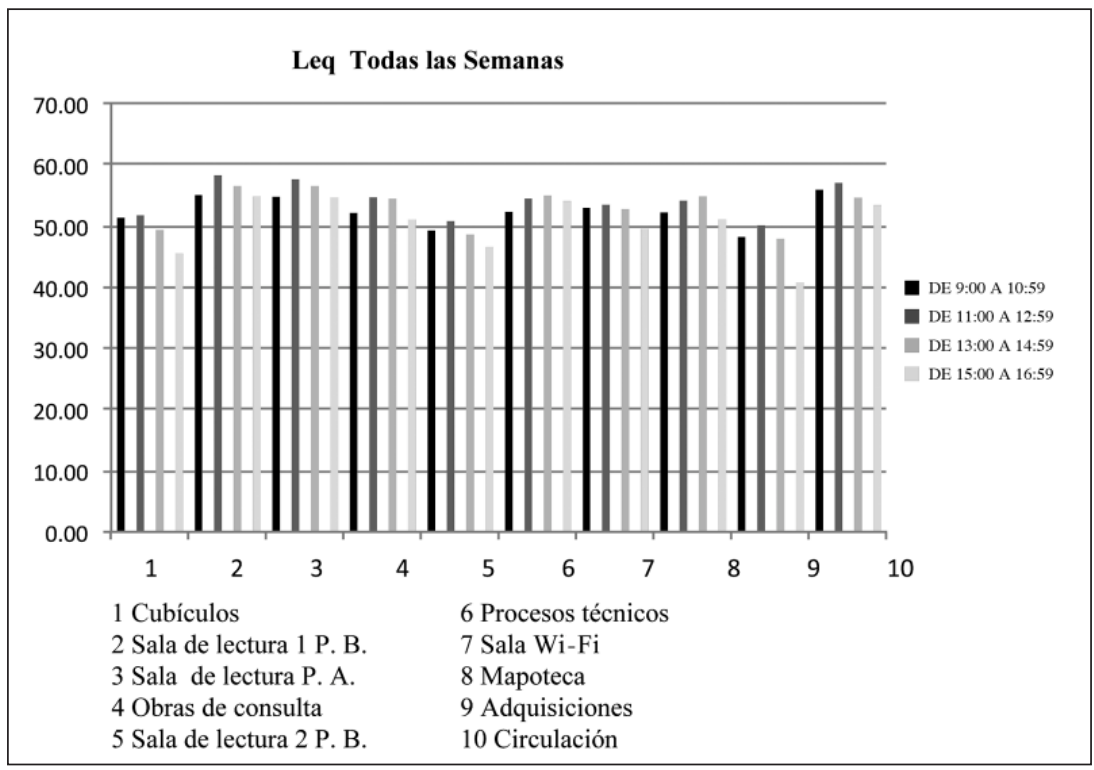

Figura 11. Nivel sonoro continuo equivalente, en rangos horarios y durante todas las semanas

La Figura 11, sin distinguir las semanas de monitoreo, da cuenta de un comportamiento relativamente homogéneo, con tendencia a tener los niveles de ruido más altos que van desde los 48 hasta los 58 dBA en los horarios inter- 
medios, es decir, de 11:00 a 12:59 y de 13:00 a 14:59; en cambio, los horarios de 9:00 a 10:59 y de 15:00 a 16:59 registraron los niveles de ruido más bajos.

En la Figura 12 destacan las zonas más ruidosas encontradas a lo largo del estudio: la sala de lectura 1 planta baja, la sala de lectura planta alta y la zona de circulación; por su parte, la sala de lectura 2 planta baja y adquisiciones son las que reportaron niveles de ruido más satisfactorios para un recinto bibliotecario.

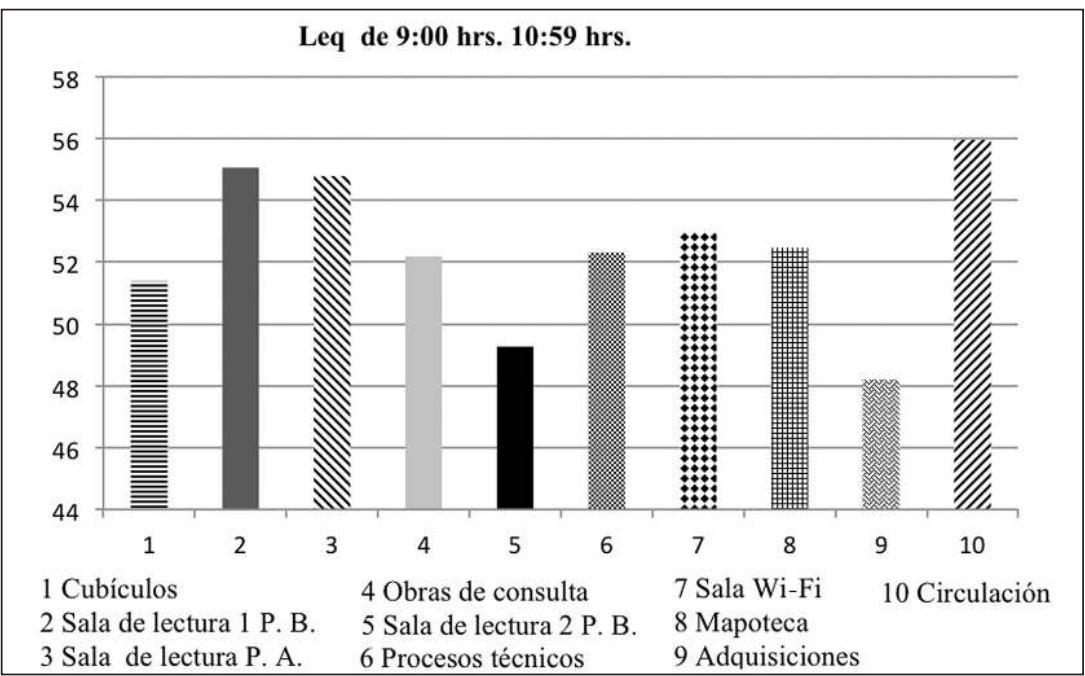

Figura 12. Nivel sonoro continuo equivalente en las diferentes áreas con relación al horario de 9:00 a 11:00

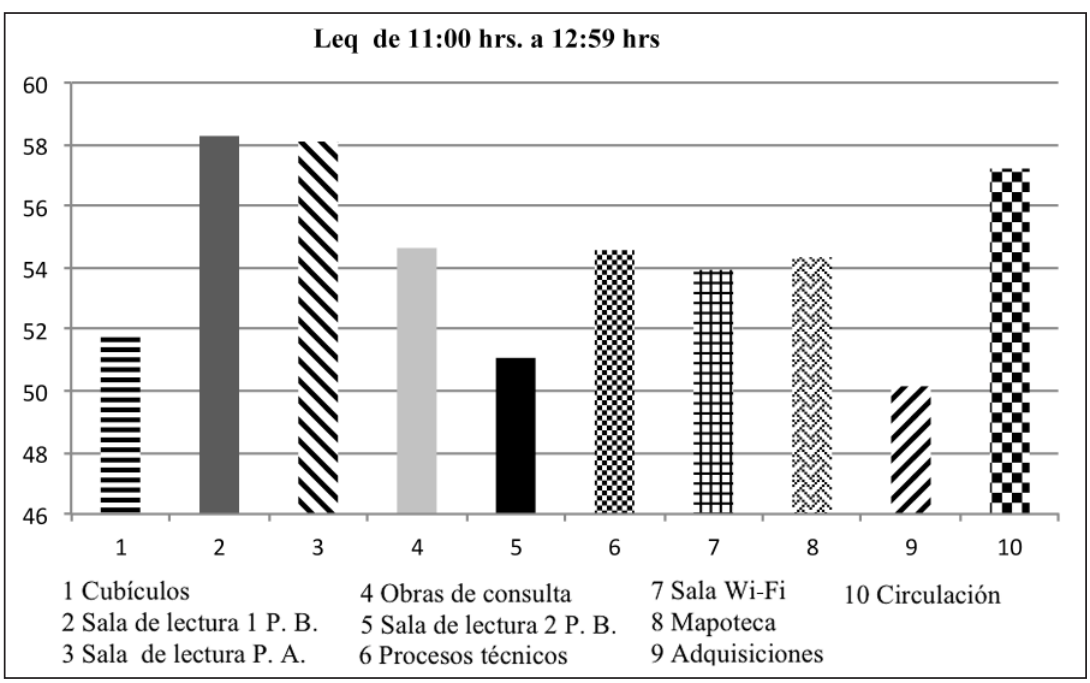

Figura 13. Nivel sonoro continuo equivalente en las diferentes áreas con relación al horario de 11:00 a 13:00 
Destacan los niveles tranquilos en cubículos, sala de lectura 2 planta baja y el área de adquisiciones, representados en la Figura 13, en los registros de este horario. La condición arquitectónica del área de adquisiciones ayuda a que se atenúen los niveles. Sobresalen como ruidosas en este horario las áreas 2,3 y 10 , las cuales se comportaron de manera relativamente constante a lo largo de este estudio.

Finalmente, en la Figura 14, los niveles que se estudiaron y determinaron en este horario dentro del recinto llaman la atención por ser más homogéneos que en los anteriores, y en la mayoría, entre los 48 y $57 \mathrm{dBA}$. Los niveles que sobresalen de nuevo son las salas de lectura 1 planta baja, la sala de lectura planta alta y el área de circulación.

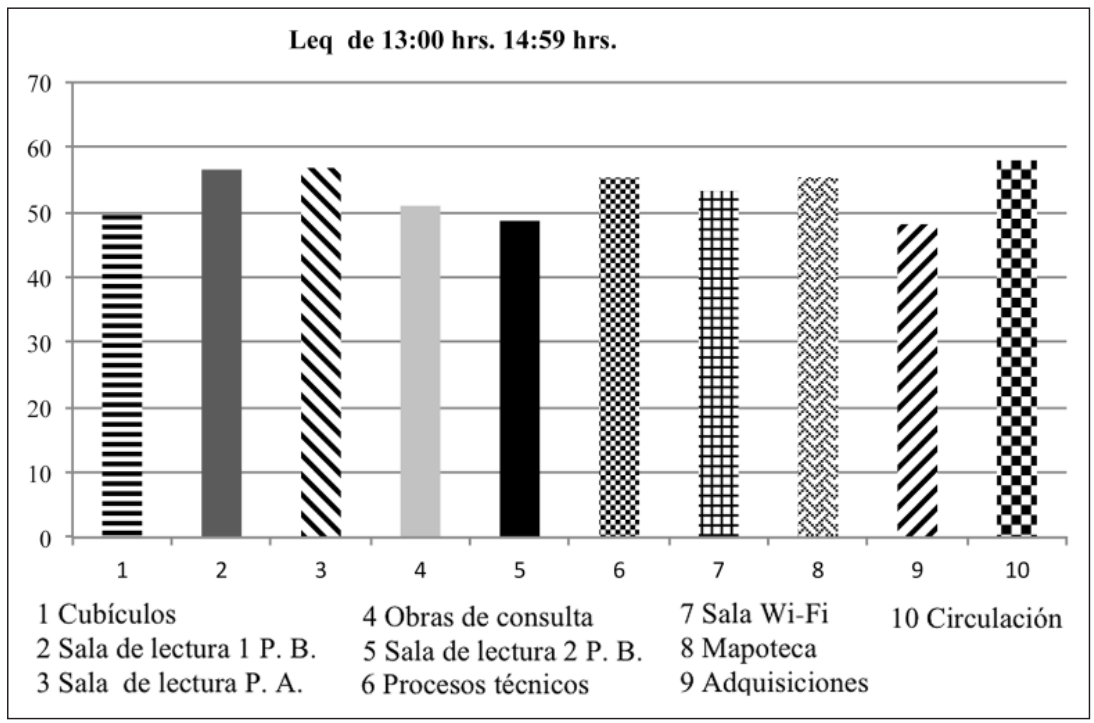

Figura 14. Nivel sonoro continuo equivalente en las diferentes áreas con relación al horario de 13:00 a 15:00

Es importante señalar que dentro de los ruidos encontrados los más significativos se dieron cuando el usuario ingresa o sale de la biblioteca, cuando las impresoras y fotocopiadoras están funcionando a elevado volumen e intensidad, los teclados de computadoras, las conversaciones entre dos, tres o más usuarios, cuando suenan los teléfonos celulares, con el movimientos de sillas, cuando los tacones son demasiado molestos y cuando la condición arquitectónica no favorece la atenuación; éstos son algunos de los elementos que provocan que el nivel de ruido en la biblioteca del CUCBA no sea deseable.

El estudio de Ntui (2009) acerca de las fuentes y niveles de ruido en una biblioteca de Calabar, Nigeria, reporta la presencia de ruido; sin embargo, exis- 
ten diferencias en virtud de que la biblioteca estudiada se encuentra alejada del ruido vehicular; en cambio, los ruidos generados por los usuarios y las tecnologías existentes en las bibliotecas se reportan en ambos casos como presentes.

\section{CONCLUSIONES Y RECOMENDACIONES}

Con base en los objetivos planteados y los resultados generados, queda de manifiesto la contribución al ruido por parte de los usuarios en el área de ingreso y salida de la biblioteca, además de que los aparatos y equipos contribuyen también al deterioro de la calidad acústica, así como fuentes externas como los aviones y las propias características del diseño arquitectónico.

Se registraron datos que sobrepasan los $50 \mathrm{~dB}(\mathrm{~A})$, lo cual confirma la dificultad que existe en el sitio para lograr condiciones acústicas que se ajusten a los límites máximos recomendados por la Organización Mundial de la Salud (45 dBA); destaca que la mayoría de los registros se encuentran fuera de la norma para bibliotecas e incluso para las permitidas en zonas urbanas producidas por fuentes fijas, lo que da cuenta de la afluencia de alumnos y lo inadecuado del edificio.

El ruido se incrementa en áreas como las salas de lectura, la de obras de consulta, la mapoteca y la de circulación, por motivos de la propia actividad y la presencia de los estudiantes. Es importante tomar en consideración que la actividad propia de los universitarios dificulta que se consigan condiciones de menor nivel de ruido, sobre todo debido a la relación con las características físicas del recinto y del cristal, que potencia los niveles de ruido.

Los niveles máximos en fin de semestre superan los $60 \mathrm{dBA}$ y en inicio de clases llegan hasta 70 dBA. Los niveles mínimos durante la semana en fin de semestre rondan los $50 \mathrm{dBA}$. El horario en el que se incrementan los niveles suele ser de las 11:00 a las 12:59 y el más tranquilo va de las 15:00 a las 16:59 hasta con 15 decibeles menos, en un nivel sonoro continuo equivalente.

Dadas las condiciones que se registraron, se considera importante incorporar medidas de atención que permitan una condición acústica más adecuada para la biblioteca, lo cual debe incluir difusión con carteles, conferencias e información para los usuarios, puesto que es evidente que los niveles de ruido registrados en la biblioteca son elevados para el tipo de actividad que ahí se realiza.

En la medida en que las bibliotecas universitarias sean capaces de realizar sus funciones e incluyan dentro de sus factores de confort y bienestar un ambiente de aprendizaje tranquilo, el entorno para el usuario será más favorable.

Una propuesta que se identifica como valiosa es la incorporación de una normatividad y legislación mexicana para la construcción de edificios que to- 
me en consideración proyectos de investigación como el que aquí se presenta y la experiencia generada en otros países.

Para la construcción de edificios destinados a albergar una biblioteca es indispensable considerar los aportes que el bibliotecario puede emitir en relación con las áreas y secciones, ya que puede señalar las características específicas necesarias, al igual que los espacios escolares que requieren estudios más sistemáticos.

Las colecciones consideradas de mayor demanda deben estar ahí donde sea posible tener la mayor vigilancia y control, con el objeto de poder influir en la atenuación del ruido.

La planificación del edificio de la biblioteca central estudiada no contempló los elementos esenciales que la biblioteca universitaria debe tener en su diseño arquitectónico para las colecciones, los servicios, las salas de lectura y el crecimiento y la flexibilidad que deben guardar las áreas del personal y la administración, cuidando la interrelación para el desarrollo de las actividades técnicas de la biblioteca, como se ha descrito ya en este trabajo.

Las condiciones geográficas de una zona rural, como es el caso del edificio de esta biblioteca, donde se utilizó material de construcción que permite el acceso de polvo, plagas, agua y radiación solar, hacen vulnerables los materiales impresos y obligan a reubicarlos o bien a incluir filtros solares y antireflejantes que minimicen el ingreso de luz.

El diseño en la construcción del edificio con tendencia modernista omitió consideraciones de aislamiento para el ruido como sucede en el área de cubículos y la sala Wi-Fi, que dejaron espacios muertos de construcción debido a las columnas de hierro y la presencia de una escalera central que limitan la visibilidad y el reacomodo del acervo o de la sala de lectura.

Los espacios destinados para los usuarios, los acervos y el personal del edificio estudiado ponen de manifiesto la ausencia de un perito arquitecto en el desarrollo de proyectos arquitectónicos para bibliotecas universitarias. Tampoco se contempló la aireación en los baños del personal ni un espacio para lavar los utensilios de limpieza.

Los estudios de calidad ambiental en bibliotecas y en particular de contaminación por ruido deben incluir una estrategia universitaria que contribuya a dotar mejor los espacios para el aprendizaje y la actividad académica, con los beneficios que esto supone.

La presente investigación deja abierta la posibilidad de continuar realizando proyectos similares en otras instancias, así como también la oportunidad de profundizar en la percepción de las condiciones acústicas que encuentran los usuarios de la biblioteca. 


\section{BiBLIOGRAFÍA}

Abad, L. T. et al. (2011), "Ruido ambiental, seguridad y salud", en Revista de Ciencia y Tecnología y Medio Ambiente, vol. VIII, Separata, Universidad Alfonso X El Sabio, Escuela Politécnica Superior Villanueva de la Cañada (Madrid), pp. 1-24.

Baigorri, A. (1995), "Apuntes para una Sociología del Ruido", conferencia presentada en $V$ Congreso Español de Sociología, Granada, España, Federación Española de Municipios y Provincias, 28 al 30 de septiembre.

Bau, G. P. (2011), Estudio de los factores físico-ambientales y su influencia en la percepción del confort de los usuarios de la biblioteca de informática (UPV), tesis de licenciatura, Valencia, Universidad Politécnica de Valencia, 129 pp.

Berglund, B.; Lindvall, T. y Schwella, D. (1999), Guidelines for Community Noise. World Health Organization WHO, Londres, Inglaterra, 159 pp.

Beristain, S. (1998), “Ruido”, en Segio Beristain et al. (coord.), Memorias del V Congreso Mexicano de Acústica (V Congreso Mexicano de Acústica, Instituto Mexicano de Acústica, 17 y 18 de septiembre de 1998, Querétaro), México, Instituto Mexicano de Acústica, pp. 207-214.

Bisbrouck, M. F. (2003), "Libraries as places: buildings for the 21st century", en Proceedings of the thirteenth seminar of IFLA's library buildinsg and equipement section, París, Francia, 210 pp.

Carrión, I. A. (1998), Diseño acústico de espacios arquitectónicos, Barcelona, Ediciones de la Universidad Politécnica de Catalunya, 433 pp.

Chávez, A. M. (1998), Asociación entre el ruido ambiental y capacidad auditiva en voceadores de la zona centro de la ciudad de Guadalaja$r a$, tesis de maestría, México, Universidad de Guadalajara, Centro Universitario de Ciencias Biológicas y Agropecuarias, 108 pp.

Correa, S. F.; Osorio, M. J. D. y Patiño, V. B. (2011), "Valoración económica del ruido: una revisión analítica de estudios", en Semestre Económico, 4 (29), pp. 53-76.

Crumpton, M. A. (2007), "Sounding Off About Noise”, en Community EJunior College Libraries, 13 (4), pp. 89-99.

De Esteban, A. A. (2003), "Contaminación acústica y salud", en $O b$ servatorio medioambiental, 6, pp. 73-95.

Delgadillo, S. A. (1998). Contribución al estudio de ruido ambiental, fundamentos teóricos y problemas en la zona centro de la ciudad de Guadalajara, tesis de licenciatura, México, Universidad de Guadalajara, Centro Universitario de Ciencias Biológicas y Agropecuarias, $141 \mathrm{pp}$.

Estándares para bibliotecas universitarias chilenas (2001), Santiago, Chile, Consejo de Rectores de Universidades Chilenas, Comisión Asesora de Bibliotecas y Documentación. 
Estrada, G. R. F. (2010), "La enseñanza de la física y las matemáticas: un enfoque hacia la educación ambiental", en Latin American Journal of Physics Education, 4 (2), pp. 435-440.

Fuentes, R. J. J. (2005), "El edificio", en O. L. Orera (coord.), La biblioteca universitaria: análisis en su entorno bíbrido, Madrid, Síntesis; pp. 51-82.

García, A. (1994), "Algunas consideraciones sobre la contaminación acústica en zonas urbanas”, en Armando Calvo et al. (coord.), Tecniacústica 94 (Jornadas Nacionales de Acústica. Universidad de Valencia y Sociedad Española de Acústica, 15 al 17 de noviembre de 1994, Valencia), Valencia, Sociedad Española de Acústica, pp. XV-XXVIII.

García, S. y Garrido, F. (2003), La contaminación acústica en nuestras ciudades, Barcelona, Fundación "La Caixa”, 252 pp.

German, G. M. y Santillán, A. O. (2006), "Del concepto de ruido urbano al de paisaje sonoro”, en Bitácora, 10 (1), pp. 39-52.

Gisbert, M. y Esteve, F. (2011), "Digital Learners: la competencia digital de los estudiantes universitarios”, en La Cuestión Universitaria, 7, pp. 48-59.

Gómez H., J. A. (2002), Gestión de Bibliotecas, Murcia. Disponible en: http://www.um.es/gtiweb/jgomez/bibgen/intranet/07edificio. PDF [Fecha de consulta: 24 de enero del 2013].

López, U. et al. (2000), "Hipoacusia por ruido: un problema de salud y conciencia pública”, en Revista de la Facultad de Medicina, Universidad Nacional Autónoma de México, 43 (2), pp. 41-42.

López, G. L. (s. a.), ¿Normalización de ruido en bibliotecas?, Instituto Tecnológico de Ciudad Juárez.

Martínez, D. (2004), "El Centro de recursos para el aprendizaje e investigación: un nuevo modelo de biblioteca para el siglo XXI", en Educación y Biblioteca, pp. 98-108.

Martínez S., A. (2005), "Ruido por tráfico urbano: conceptos, medidas descriptivas y valoración económica”, en Economía y Administración, s/n., pp. 1-49.

Mosca, D. (1980), Contaminación Acústica Originada por el Ruido de Tránsito y Proposiciones para su Evaluación en el Área Metropolitana. Ingeniero Técnico, tesis de grado, Ingeniero Técnico, Santiago, Universidad de Chile, Departamento de Obras Civiles, 116 pp.

Muñoz, C. A. (2004), Los espacios del saber: bistoria de la arquitectura de las bibliotecas, Gijón, Trea, 397 pp. (Colección Biblioteconomía y Administración Cultural, 94)

Norma Oficial Mexicana NOM-081-SEMARNAT-1994. Que establece los límites máximos permisibles de emisión de ruido de las fuentes fijas y su método de medición, 18 pp.

Normas para bibliotecas de instituciones de educación superior e investigación (2012), México, Consejo Nacional para Asuntos Bibliotecarios de las Instituciones de Educación Superior, 52 pp. 
Ntui, A. I. (2009), "Noise Sources and levels at the University of Calabar Library, Calabar, Nigeria”, en African Journal of Library, Archives and Information Science, 19 (1), pp. 53-63.

Orozco, M. G. (2008), "El análisis de ruido en Guadalajara: Elementos Clave para la realización de estudios de ruido urbano. Capítulo 6”, en A. Curiel-Ballesteros (ed.), Investigación Socioambiental: Paradigmas aplicados en salud ambiental y educación ambiental, México, Universidad de Guadalajara, pp. 161-182.

— y González, A. (2012) (coords.), Ruido en ciudades latinoamericanas: bases para su gestión, Guadalajara, Universidad de Guadalajara, $206 \mathrm{pp}$.

Palafox, O. (2003), Análisis de un problema de calidad ambiental por niveles de ruido presentes en la colonia Auditorio (octubre, 2001-2002) Zapopan, tesis licenciatura, México, Universidad de Guadalajara, Centro Universitario de Ciencias Biológicas y Agropecuarias, 122 pp.

Prensky, M. (2001a), "Digital natives, digital immigrants", en The Horizon, 9 (5), pp 1-6.

(2001b), “The digital game-based learning revolution", en Digital Game-Based Learning, pp 1-19.

Quimbiulco, Ch. D. A. (2008), Diseño e implementación de un prototipo que permita cuantificar el nivel de presión sonora en una biblioteca con indicación visual y audible, tesis de licenciatura, Ecuador, Escuela Politécnica Nacional, Facultad de Ingeniería Eléctrica y Electrónica, 137 pp.

Quintero, T. A. y Muñoz, A. Y. (2009), "Protección jurídica frente al ruido. Los instrumentos regulatorios administrativos", en Revista Ámbito Jurídico Rio Grande, 12 (65). Disponible en: http://www.ambi to-juridico.com.br/site/?n_link=revista_artigos_leitura\&artigo_id $=6128 \&$ revista_caderno $=4$ [Fecha de consulta: 8 de marzo de 2013].

Rodríguez G., A. (1996) (coord.), Nuevos edificios para las bibliotecas universitarias, México, UNAM-Dirección General de Bibliotecas, 174 pp.

Sanz, S. J. M. (1990), El Ruido, Madrid, Ministerio de Obras Públicas y Urbanismo, Centro de Publicaciones, 132 pp.

Soriano, L. (1995), “Contaminación por ruido ambiental”, en Sergio Beristain et al. (coord.), Memorias del Segundo Congreso Mexicano de Acústica (Congreso Mexicano de Acústica, Segundo, Instituto Mexicano de Acústica, 21 y 22 de septiembre de 1995, Guadalajara), México, Instituto Mexicano de Acústica, pp. 59-72.

Tapscott, D. (1998), Growing up digital: The rise of the net generation, Nueva York, McGraw-Hill, 338 pp.

— y Williams, A. D. (2009), Wikinomics: How mass collaboration changes everything, Nueva York, Penguin Group, 357 pp.

Villalobos, L. y Elizondo, G. (1999), "Ruido producido por peregrinaciones religiosas en la zona urbana de Monterrey", en Ingenierías, 2 (4), pp 11-17. 